\title{
Molecular Origin of Anomalous pH Effect in Blue Proteorhodopsin
}

\author{
Mizuki Sumikawa, ${ }^{\dagger}$ Rei Abe-Yoshizumi,${ }^{\dagger}$ Takayuki Uchihashi,,+ and Hideki Kandori ${ }^{*}, \dagger, \#$
}

†Department of Life Science and Applied Chemistry, Nagoya Institute of Technology, Showa-ku, Nagoya 466-8555, Japan

Department of Physics, Nagoya University, Nagoya 464-8602, Japan.

\#OptoBioTechnology Research Center, Nagoya Institute of Technology, Showa-ku, Nagoya 466-8555, Japan

Email: kandori@nitech.ac.jp

\section{Methods:}

Preparation of VcBPR Sample. By searching homologous proteins to blue proteorhodopsin (BPR) from marine $\gamma$-proteobacteria Photobacterium angustum LC1-200, ${ }^{1}$ we found BPR from Vibrio califitulae (VcBPR). The amino acid identity is 95\% between BPR in LC1-200 and VcBPR. The gene of VcBPR was synthesized and cloned to pET21 vector possessing a sixhistidine tag at the C-terminus. The expression plasmid, pET-VcBPR was transformed in E. coli strain BL21 (DE3) (Stratagene), solubilized with 1\% n-dodecyl $\beta$-D-maltoside (DDM), and purified via $\mathrm{Co}^{2+}$ column chromatography as described previously. ${ }^{1}$

UV-Visible Spectroscopy. Absorption spectra were recorded for solubilized PR (0.03\% DM, $150 \mathrm{mM} \mathrm{NaCl}$, and $10 \mathrm{mM}$ citric acid monohydrate, MES, MOPS, HEPES, CHES and CAPS) by using a UV2450 visible spectrophotometer (Shimadzu Corp.). ${ }^{1}$ The initial volume and $\mathrm{pH}$ were 5 $\mathrm{ml}$ and 7 , from which $\mathrm{pH}$ was increased to 10 by addition of $\mathrm{NaOH}$. Then, $\mathrm{pH}$ was decreased to 2 by addition of $\mathrm{HCl}$, where the absorption spectrum was measured every $0.5 \mathrm{pH}$ unit. We confirmed that absorption spectra at the same $\mathrm{pH}$ produced by additions of either $\mathrm{NaOH}$ or $\mathrm{HCl}$ were identical, indicating that $\mathrm{pH}$ titration was reversible between $\mathrm{pH} 4$ and 7 . The $\lambda_{\max }$ was plotted as a function of $\mathrm{pH}$, and the Henderson-Hasselbalch equation was used for determination of the $\mathrm{p} K_{\mathrm{a}}$ values. 
Size-Exclusion Chromatography (SEC). Samples containing about purified VcBPR (OD at $\left.\lambda_{\max }=0.2\right)$ suspended in the same buffer $(\mathrm{pH} 7.0)$ described above were applied to a Superdex 200 5/150 GL size-exclusion column (GE Healthcare U.K., Amersham Place, England) which had been previously equilibrated with the same buffer. All SEC experiments were carried out at $4{ }^{\circ} \mathrm{C}$. The protein absorption was simultaneously monitored at four different wavelengths, 230 , 280,495 , and $540 \mathrm{~nm}$.

High-speed AFM. To observe the oligomric states before and after the acidic treatment of VcBPR, a laboratory-built high-speed AFM operated in tapping mode was employed. ${ }^{2,3}$ The typical physical properties of the cantilever used in this study were a spring of $0.2 \mathrm{~N} / \mathrm{m}$, a resonant frequency of $\sim 700 \mathrm{kHz}$, and quality factor of $\sim 2$ in a liquid condition. As an AFM probe, amorphous pilar was prepared by electron beam depositon and then sharpened to be the end radius less than $2 \mathrm{~nm}$ by Ar plasma etching. Samples for AFM observation were prepared in the same way as previously reported, with the following steps. ${ }^{4}$ The VcBPR dissolved in the buffer including $0.1 \%$-DM was deposited on a freshly cleaved mica and after $3 \mathrm{~min}$ of incubation, washed with the same buffer to remove residual molecules. Next, lipid membranes from E. coli (Avanti Polar Lipids Inc. dissolved in buffer containing 0.1\%-DM $)(0.1 \mathrm{mg} / \mathrm{ml})$ were placed on the mica substrate and washed with buffer without DM after 10 min of incubation. Finally the HS-AFM observation was carried out in a buffer solution $(20 \mathrm{mM}$ Tris- $\mathrm{HCl}, \mathrm{pH} 8.0$ and $100 \mathrm{mM}$ $\mathrm{NaCl})$ at room temperature. 


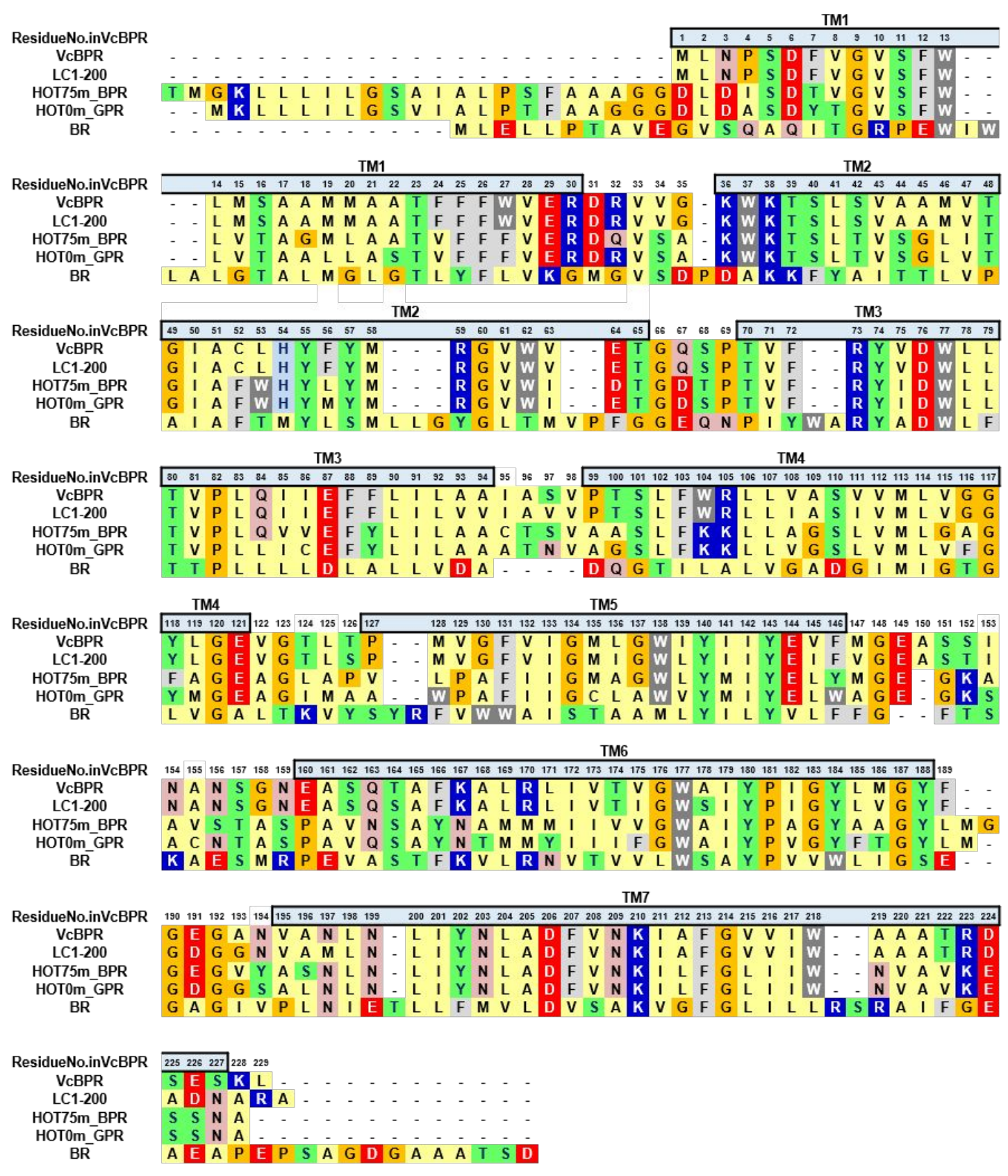

Figure S1. Sequence alignment of selected microbial rhodopsins. The alignment of amino acid sequences of VcBPR (blue-absorbing proteorhodopsin from Vibrio califitulae), LC1-200 (blueabsorbing proteorhodopsin in LC1-200), ${ }^{1}$ HOT75m_BPR (blue-absorbing proteorhodospin), ${ }^{5}$ HOT0m_GPR (green-absorbing proteorhodopsin), ${ }^{5}$ and BR (bacteriorhodopsin). The positions of transmembrane helices are indicated by light blue rectangles for BPR according to the X-ray crystallographic structure (PDB ID: 4KLY). ${ }^{6}$ 


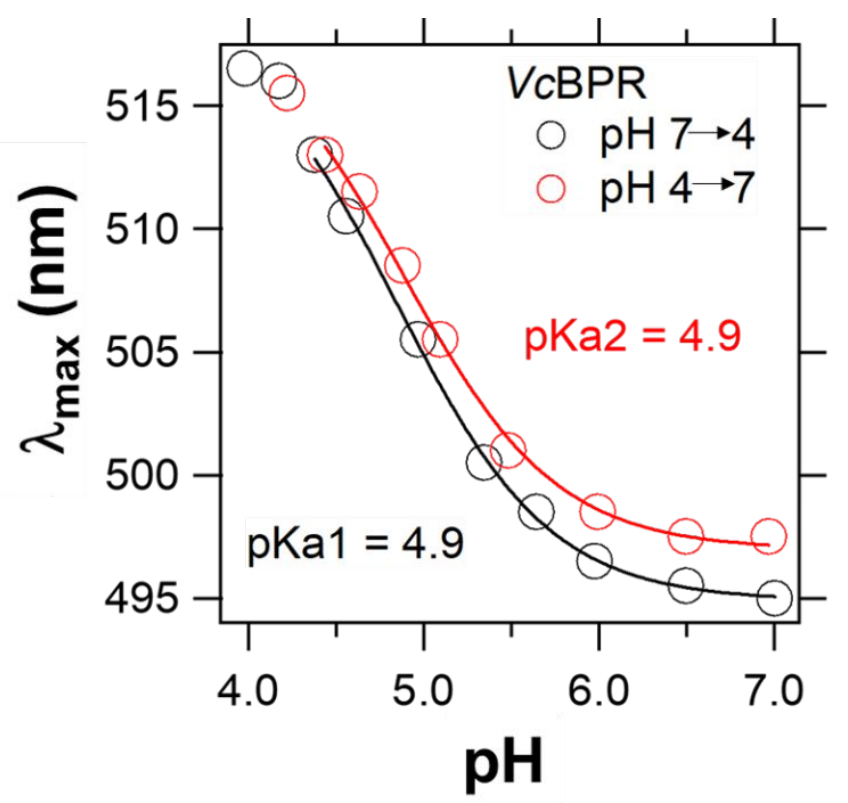

Figure S2. pH titration of VcBPR between pH 4 and 7. Black and red circles represent $\lambda_{\max }$ of VcBPR when $\mathrm{pH}$ is changed from 7 to 4 , and 4 to 7 , respectively. Black and red lines represent the pH titration curves according to the Henderson-Hasselbalch equation. 


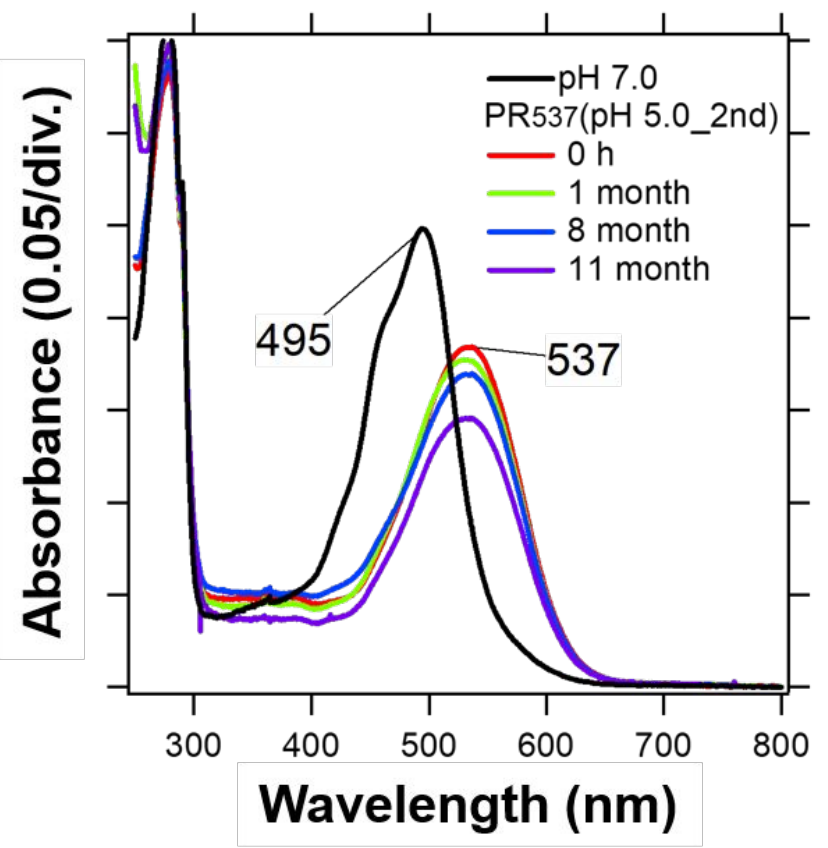

Figure S3. No reversion of $\mathbf{P R}_{537}$ to VcBPR. When $\mathrm{PR}_{537}$ is kept at $277 \mathrm{~K}$, the $\lambda_{\max }$ do not alter for 11 months. Reduction in absorbance suggests that some portion is bleached, but never returned to the original VcBPR. 
(a)

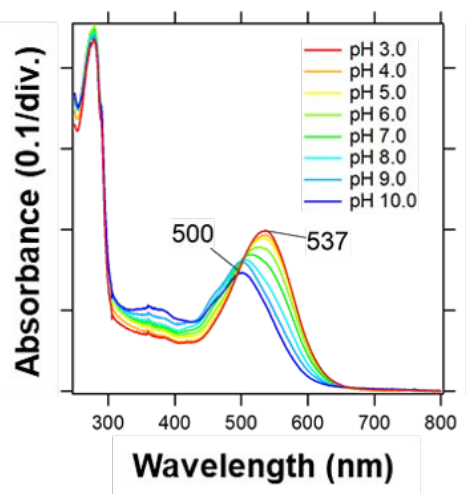

(b)

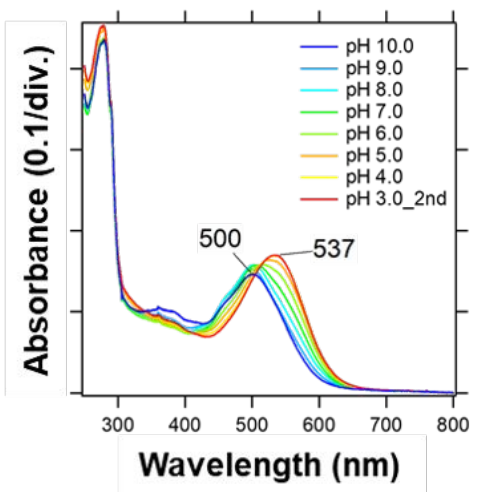

(c)

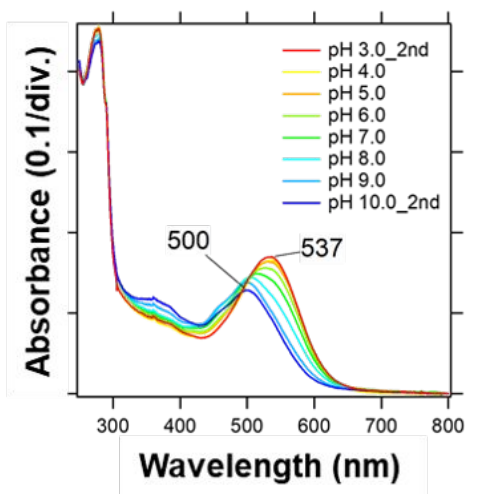

Figure S4. Absorption spectra of the $\mathbf{P R}_{537}$ species of VcBPR at various pHs. (a) Red curve represents visible absorption spectra of the $\mathrm{PR}_{537}$ species of $\mathrm{VcBPR}$ at $\mathrm{pH}$ 3.0. Then, $\mathrm{pH}$ is increased up to 10.0. The $\lambda_{\max }$ is located at 537 and $500 \mathrm{~nm}$ at pH 3.0 and 10.0, respectively. (b) Absorption spectrum at $\mathrm{pH} 10.0$ (blue line) is identical to that in (a), and $\mathrm{pH}$ is decreased to 3.0. The $\lambda_{\max }$ is located at $537 \mathrm{~nm}$ at $\mathrm{pH} \mathrm{3.0,} \mathrm{the} \mathrm{same} \mathrm{value} \mathrm{in} \mathrm{(a),} \mathrm{whereas} \mathrm{those} \mathrm{at} \mathrm{intermediate} \mathrm{pHs}$ are different (see text). (c) Absorption spectrum at $\mathrm{pH} 3.0$ (red line) is identical to that in (b), and $\mathrm{pH}$ is increased to 10.0. The $\lambda_{\max }$ at each $\mathrm{pH}$ are identical to those in (a), but not to those in (b). 


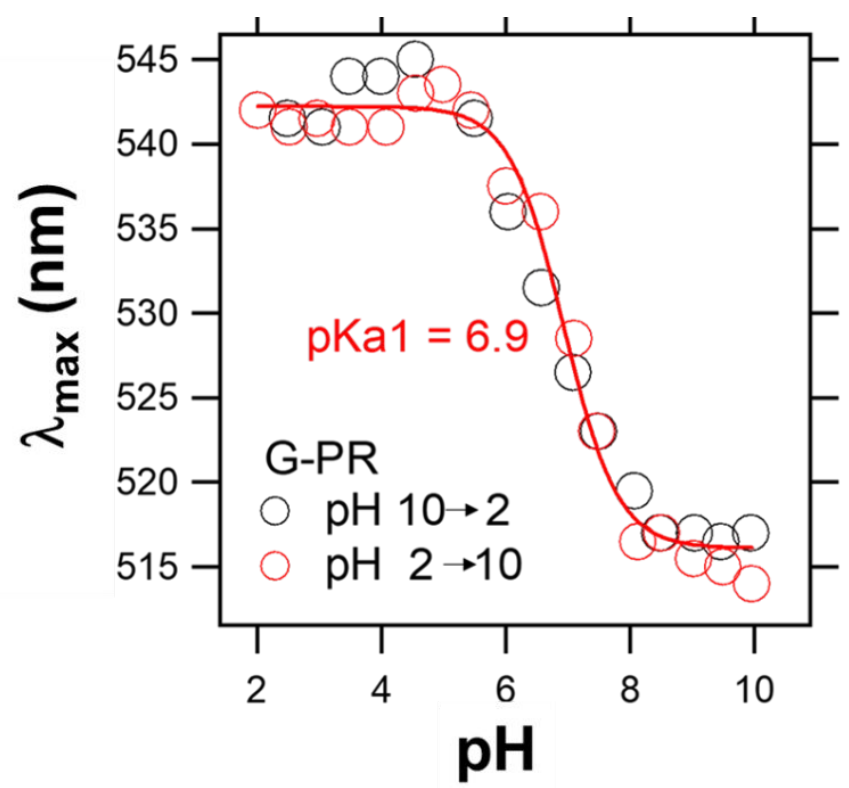

Figure S5. pH titration of GPR between pH 2 and 10. Black and red circles represent $\lambda_{\max }$ of GPR when $\mathrm{pH}$ is changed from 10 to 2 , and 2 to 10 , respectively. Red lines represents the $\mathrm{pH}$ titration curve according to the Henderson-Hasselbalch equation, whose $\mathrm{pKa}$ is determined to be 6.9. 


\section{References}

(1) Yamada, K.; Kawanabe, A.; Yoshizawa, S.; Inoue, K.; Kogure, K., Kandori, H. Anomalous pH effect of blue proteorhodopsin. J. Phys. Chem. Lett. 2012, 3, 800-804.

(2) Shibata, M.; Yamashita, H.; Uchihashi, T.; Kandori, H.; Ando, T. High-speed atomic force microscopy shows dynamic molecular processes in photo-activated bacteriorhodopsin. Nat. Nanotech. 2010, 5, 208-212.

(3) Shibata, M.; Inoue, K.; Ikeda, K.; Konno, M.; Singh, M.; Kataoka, C.; Abe-Yoshizumi, R.; Kandori, H.; Uchihashi, T. Oligomeric states of microbial rhodopsins determined by high-speed atomic force microscopy and circular dichroic spectroscopy. Sci. Rep. 2018, 8, 8262.

(4) Sumino, A.; Uchihashi, T.; Oiki, S. Oriented Reconstitution of the Full-Length KcsA Potassium Channel in a Lipid Bilayer for AFM Imaging. J. Phys. Chem. Lett. 2017, 8, 785-793.

(5) Béjà, O.; Spudich, E. N.; Spudich, J. L.; Leclerc, M.; DeLong, E. F. Proteorhodopsin Phototrophy in the Ocean. Nature 2001, 411, 786-789.

(6) Ran, T.; Ozorowski, G.; Gao, Y.; Sineshchekov, O. A.; Wang, W.; Spudich, J. L.; Luecke, H. Cross-protomer interaction with the photoactive site in oligomeric proteorhodopsin complexes. Acta Crystallogr. D Biol. Crystallogr. 2013, 69, 1965-1980. 